\title{
Glucocorticoid-associated worsening in reversible cerebral vasoconstriction syndrome
}

\section{(6)}

Aneesh B. Singhal, MD

Mehmet A. Topcuoglu, MD

Correspondence to Dr. Singhal: asinghal@partners.org

\section{ABSTRACT}

Objective: Factors predicting poor outcome in patients with the reversible cerebral vasoconstriction syndrome (RCVS) have not been identified.

Methods: In this single-center retrospective study, we analyzed the clinical, brain imaging, and angiography data in 162 patients with RCVS. Univariable and multivariable regression analysis were performed to identify predictors of persistent (nontransient) clinical worsening, radiologic worsening, early angiographic progression, and poor discharge outcome (modified Rankin Scale score 4-6).

Results: The mean age was $44 \pm 13$ years; $78 \%$ of patients were women. Persistent clinical worsening occurred in $14 \%$ at $6.6 \pm 4.1$ days after symptom onset, radiologic worsening in $27 \%$ (mainly new infarcts), and angiographic progression in 15\%. Clinical worsening correlated with angiographic progression and new nonhemorrhagic lesions. Age and sex did not independently predict any type of worsening. Infarction on baseline imaging predicted poor outcome. Prior serotonergic antidepressant use predicted clinical and angiographic worsening but not poor outcome. Intra-arterial vasodilator therapy independently predicted clinical worsening and poor discharge outcome but was offered to more severe cases. Glucocorticoid treatment proved to be an independent predictor of clinical, imaging, and angiographic worsening and poor outcome. Of the 23 patients with clinical worsening, 17 received glucocorticoids ( 15 within the preceding 2 days). There were no significant differences in baseline brain lesions and angiographic abnormalities between glucocorticoid-treated and untreated patients.

Conclusion: Patients with RCVS at risk for worsening can be identified on basis of baseline features. latrogenic factors such as glucocorticoid exposure may contribute to worsening. Neurology ${ }^{\circledR}$ 2017;88:228-236

\section{GLOSSARY}

cSAH = convexal subarachnoid hemorrhage; CTA = CT angiogram; DSA = digital subtraction cerebral angiogram; ICH = intracerebral hemorrhage; $\mathbf{M R A}=$ magnetic resonance angiogram; $\mathbf{m R S}=$ modified Rankin Scale; $\mathbf{P A C N S}=$ primary angiitis of the CNS; PRES = posterior reversible leukoencephalopathy syndrome; RCVS = reversible cerebral vasoconstriction syndrome.

Over the last decade, the clinical and imaging features of reversible cerebral vasoconstriction syndrome (RCVS) have been extensively characterized ${ }^{1-7}$ and distinguished from its historic mimic, primary angiitis of the CNS (PACNS). ${ }^{7-9}$ Patients with RCVS invariably present with recurrent severe thunderclap headaches. One-third to half develop ischemic and hemorrhagic brain lesions, either alone or in combination. Despite the dramatic onset and frequently ominous cerebral angiographic appearance, over $90 \%$ have excellent clinical outcome. ${ }^{2,6}$ Yet studies that included a high proportion of inpatients have reported poor outcome (discharge modified Rankin Scale [mRS] score $>3$, including death) in 5\%-14\% of patients. ${ }^{6,10}$

Factors predicting clinical outcome in RCVS have not been adequately identified. ${ }^{10,11}$ We have reported an association between poor clinical outcome $(\mathrm{mRS}>3)$ and glucocorticoid treatment, ${ }^{6,7}$ which if confirmed has major clinical implications since glucocorticoids are frequently offered due to consideration of PACNS, a condition that warrants prompt initiation of long-term 
immunosuppressive treatment. Other RCVS studies have shown an association between transfemoral cerebral angiography and transient worsening, ${ }^{2}$ or the development of infarcts and transient/permanent worsening. ${ }^{10}$ Vasoconstrictive drugs such as serotonergic antidepressants have been consistently identified as triggers of $\mathrm{RCVS}^{2,6,7,12}$ and may worsen vasospasm in the setting of aneurysmal subarachnoid hemorrhage $^{13,14}$; however, their contribution to RCVS-related worsening is uncertain. On the other hand, case reports have suggested a therapeutic role for calcium channel blockers and intra-arterial vasodilator infusions. ${ }^{15-17}$

We designed this study to identify predictors and mechanisms of clinical and radiologic worsening in RCVS.

METHODS Standard protocol approvals, registrations, and patient consents. This retrospective study was approved by our Institutional Human Research Committee.

We included 162 patients with RCVS encountered at Massachusetts General Hospital from 1998 to 2016. The clinical imaging features of the first 159 patients have been published. ${ }^{6,7}$ The diagnosis was established as follows: vasoconstriction reversibility was documented in 128 patients. An additional 30 patients developed typical thunderclap headaches, had evidence for segmental cerebral artery narrowing, and had a self-limited clinical course with no recurrences during follow-up. The final 4 patients had typical clinical presentations but died during hospitalization; 2 had normal cerebral arteries (no vasculitis) on autopsy and 2 had immediate angiographic resolution upon intra-arterial vasodilator infusion, consistent with RCVS.

We reviewed hospital records to extract information on demographics, triggers, medical history, neurologic deficits, daily clinical events, and laboratory results. Clinical worsening was defined as the development of new persistent focal or cognitive deficits or abrupt worsening of existing deficits. Expected evolution of baseline deficits, recurrent thunderclap headache, and transient neurologic spells (including seizures) with prompt return to baseline function were not included.

Radiologic worsening was defined as the occurrence of a new lesion on any follow-up brain scan. Lesions types were infarction, intracerebral hemorrhage ( $\mathrm{ICH})$, convexal subarachnoid hemorrhage $(\mathrm{CSAH})$, and vasogenic edema (i.e., lesions consistent with the posterior reversible leukoencephalopathy syndrome $[\mathrm{PRES}]^{18}$ ). For new infarcts and new vasogenic edema, given the low sensitivity of head CT, we used only the baseline MRI scan for comparison. We were careful to distinguish lesion progression (e.g., hematoma expansion) from new lesions.

The results of follow-up cerebral angiograms (digital subtraction cerebral angiogram [DSA], CT angiogram [CTA], and magnetic resonance angiogram [MRA], in order of preference) performed within 30 days after baseline were categorized as initially worsened, improved, or stable as compared to the immediately prior study based on the overall angiographic appearance. Angiographic categorization was completed without knowledge of treatment and prior to data analysis; moreover, the unbiased real-time clinical neuroradiology interpretations were considered for this categorization. Vasoconstriction severity scores, ${ }^{7}$ though useful for quantifying distribution and overall severity, were not used to assess progression since they are insensitive to change (e.g., caliber reduction along a greater length of the same arterial segment would not score differently).

Categorical data were analyzed using the $\chi^{2}$, Fisher exact test, Student $t$ test, or Mann-Whitney $U$ test. A value of $p<0.05$ was considered significant. Data are presented as mean (SD). Univariable analysis was performed to identify predictors of worsening. Multivariable regression models were created based on results of the univariable analysis to identify independent predictors of clinical, radiologic, and angiographic worsening and poor outcome (discharge mRS 4-6). For clinical worsening and poor outcome, to avoid overfitting due to the small number of outcome events, we created separate models for baseline factors and treatments. Odds ratios and 95\% confidence intervals were computed. Further analysis focused on whether the worsening occurred before or after exposure to identified predictors. SPSS version 21 (SPSS Inc., Chicago, IL) was used for analyses.

RESULTS The mean age was $44 \pm 13$ years, $78 \%$ women. A case example of clinical, radiologic, and angiographic worsening is shown in figure 1 .

Clinical worsening occurred in 23 patients (14\%) at an average of $6.6 \pm 4.1$ days (range 1-14 days) after onset of the first RCVS symptom, invariably thunderclap headache. As shown in table 1, patients with clinical worsening were almost all women, with higher rates of hypertension, depression, and exposure to serotonergic antidepressants. Patients who worsened had more infarcts and fewer $\mathrm{CSAH}$ on the admission scan. Clinical worsening was associated with new brain infarcts (from $44 \%$ to $70 \%$ ) on follow-up imaging, and more severe as well as more diffuse vasoconstriction as evidenced by the greater frequency of involvement of the intracranial internal carotid, anterior cerebral, and vertebro-basilar arteries. Patients with worsening received significantly higher rates of treatment with intra-arterial vasodilators and immunosuppressive agents such as cyclophosphamide and glucocorticoids. They had a significantly longer duration of hospitalization and worse discharge outcomes.

Radiologic worsening occurred in 44 patients (27\%) (table 2). New lesions comprised isolated infarctions in 19 patients, isolated $\mathrm{CSAH}$ in 8 , isolated vasogenic edema in 4, isolated ICH in 2, and lesion combinations in 11. Overall, new infarctions were observed in 27 patients (61.3\%) with radiologic worsening. The group with radiologic worsening was significantly older by an average of 5 years, and had more women and Hispanics. As with the clinical worsening group, they had significantly more infarctions on admission scans, accumulated more infarctions over time, showed more severe and more diffuse vasoconstriction, received more treatment, and had a longer length of stay and worse discharge outcomes. These findings were mainly driven by the subset of 27 patients with new infarctions (table 2).

In order to investigate mechanisms underlying clinical and radiologic worsening, we analyzed angiographic changes. The mean time from symptom onset 

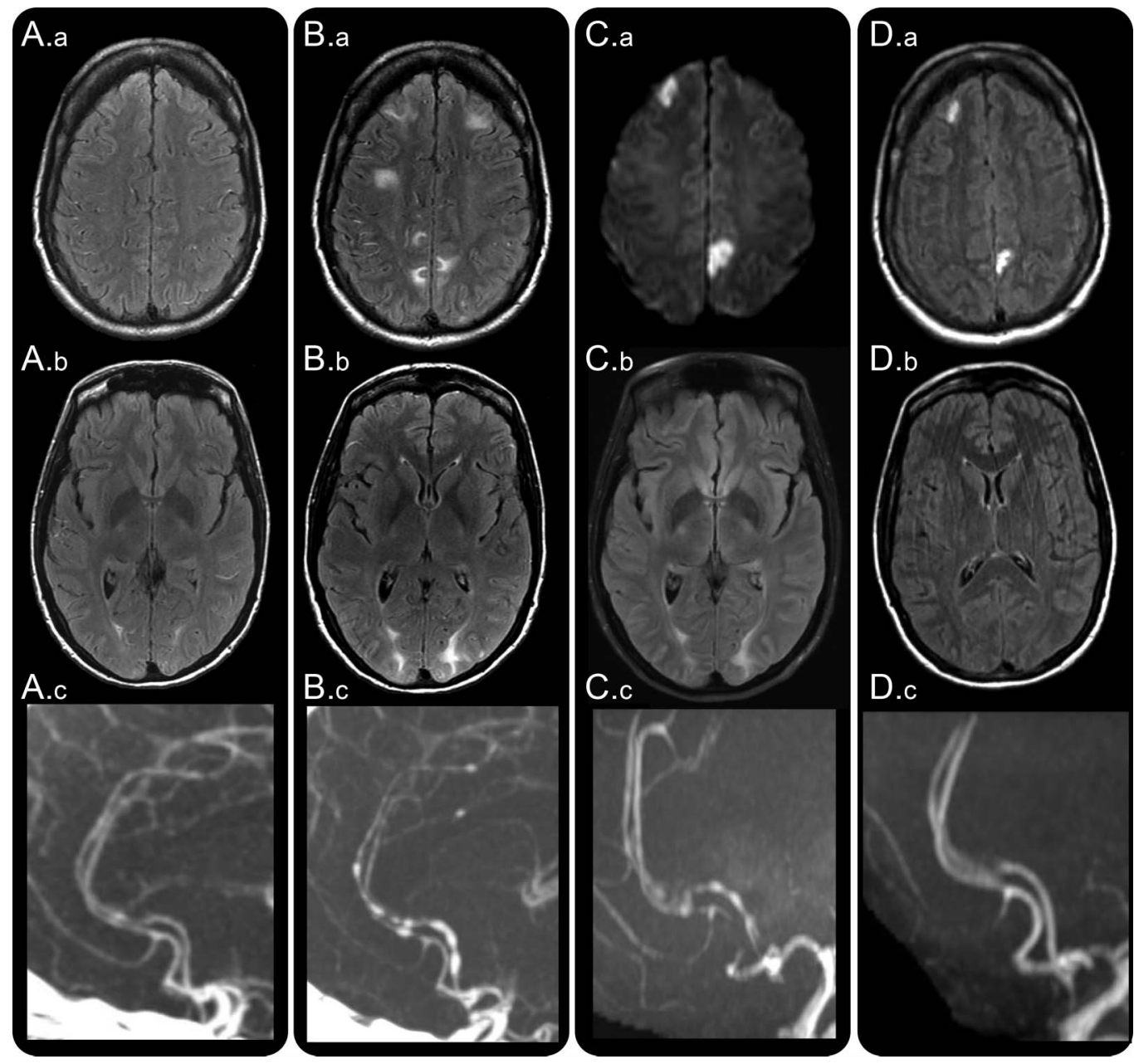

A 43-year-old woman with malabsorption syndrome, on parental nutrition, was hospitalized for treatment of port-a-cath infection. On day 2, she developed a thunderclap headache. Head CT and CSF examination were normal. Hydrocortisone $100 \mathrm{mg}$ three times daily was administered for suspected adrenal insufficiency and sepsis. On day 4, brain MRI fluid-attenuated inversion recovery (FLAIR) images showed multiple dot and linear shaped sulcal hyperintensities suggesting dilated cortical surface arteries (A.a) and subtle bilateral white matter vasogenic edematous lesions (A.b) consistent with posterior reversible encephalopathy syndrome (PRES). Headaches recurred, and on day 7 she developed cortical blindness. Head CT angiography (A.C) showed segmental narrowing of multiple intracranial arteries. Brain MRI showed new PRES lesions and progression of prior PRES lesions (B.a, B.b). She was transferred to our hospital. Neurologic examination showed features of the Balint syndrome and aphasia. Repeat CT angiography showed worsening cerebral vasoconstriction (B.C). Hydrocortisone was tapered to $25 \mathrm{mg}$ twice daily; nimodipine and magnesium were administered for suspected RCVS. Repeat MRI on day 9 showed new bilateral ischemic lesions on diffusion-weighted images (C.a), persistent PRES (C.b), and stable cerebral vasoconstriction (C.c). The port-a-cath infection was successfully treated. She was discharged on day 19 on oral prednisone $5 \mathrm{mg}$ daily and nimodipine. Follow-up imaging on day 42 showed established infarctions on FLAIR images (D.a), reversal of PRES lesions (D.b), and resolution of vasoconstriction (D.c). Neurologic examination showed no residual deficits.

to first cerebral angiography was $2.8 \pm 0.7$ days. A total of 62 patients (41\%) underwent DSA, the rest CTA/MRA. To investigate angiographic progression, we analyzed serial angiographic studies performed within the first month after onset. A total of 110 $(68 \%)$ patients underwent 462 angiograms within 30 days. Of these, 25 (15.4\%) showed initial progression and 24 (15\%) showed early reversal of vasoconstriction. No significant change in the overall angiographic appearance was seen in $61(38 \%) \mathrm{pa}-$ tients; however, 19 showed involvement of different arterial segments on follow-up, consistent with the dynamic nature of angiographic changes in RCVS. As compared to patients with stable or improving angiographic results, patients with angiographic worsening had significantly higher rates of clinical worsening ( $44 \%$ vs $12 \%, p<0.001)$, new brain lesions ( $52 \%$ vs $24 \%, p=0.005)$, new infarctions ( $32 \%$ vs $14 \%, p=0.032)$, and new vasogenic edema (20\% vs $4 \%, p=0.01)$, but not ICH ( $8 \%$ vs $3 \%, p=0.24)$ or cSAH $(16 \%$ vs $8 \%, p=0.18)$. Patients with angiographic worsening had significantly higher rates 
Table 1 Clinical worsening in reversible cerebral vasoconstriction syndrome

\begin{tabular}{|c|c|c|c|}
\hline Variable & $\begin{array}{l}\text { Worsened } \\
(n=23)\end{array}$ & $\begin{array}{l}\text { Not worsened } \\
(n=139)\end{array}$ & $p$ Value \\
\hline Age, y & $46 \pm 10$ & $43 \pm 13$ & 0.296 \\
\hline Female & 96 & 75 & $0.026^{a}$ \\
\hline Caucasian race & 74 & 85 & 0.299 \\
\hline Prior hypertension & 61 & 33 & $0.011^{a}$ \\
\hline Prior depression & 61 & 38 & $0.040^{a}$ \\
\hline Prior migraine & 48 & 40 & 0.455 \\
\hline Serotonergic antidepressants & 61 & 30 & $0.004^{a}$ \\
\hline Illicit drugs & 17 & 21 & 0.702 \\
\hline Postpartum state & 9 & 10 & 0.838 \\
\hline Idiopathic & 26 & 30 & 0.688 \\
\hline Recurrent thunderclap headaches & 70 & 75 & 0.594 \\
\hline Focal neurologic deficits & 87 & 32 & $<0.001^{a}$ \\
\hline \multicolumn{4}{|l|}{ Initial imaging } \\
\hline No lesion & 26 & 31 & 0.639 \\
\hline Infarct & 44 & 24 & $0.047^{a}$ \\
\hline Parenchymal hemorrhage & 22 & 10 & 0.107 \\
\hline Convexal SAH & 13 & 37 & $0.022^{a}$ \\
\hline Vasogenic edema (PRES) & 22 & 27 & 0.621 \\
\hline
\end{tabular}

Final imaging

\begin{tabular}{lllc} 
No lesion & 4 & 27 & $0.012^{\mathrm{a}}$ \\
Infarct & 70 & 27 & $<0.001^{\mathrm{a}}$ \\
\hline Parenchymal hemorrhage & 26 & 11 & $0.043^{\mathrm{a}}$ \\
\hline Convexal SAH & 22 & 41 & 0.078 \\
Vasogenic edema (PRES) & 22 & 29 & 0.485 \\
Vasoconstriction severity score & $1.00 \pm 0.56$ & $0.63 \pm 0.44$ & $0.001^{\mathrm{a}}$ \\
\hline Intracranial ICA involved & 35 & 13 & $0.015^{\mathrm{a}}$ \\
\hline Middle cerebral artery involved & 87 & 89 & 0.769 \\
Anterior cerebral artery involved & 100 & 82 & $0.026^{\mathrm{a}}$ \\
\hline Posterior cerebral artery involved & 91 & 77 & 0.126 \\
Vertebral or basilar arteries involved & 70 & 45 & $0.026^{\mathrm{a}}$ \\
\hline Calcium channel blocker therapy & 70 & 53 & 0.108 \\
Immunosuppressive therapy & 13 & 4 & 0.087 \\
\hline Glucocorticoid use & 74 & 21 & $<0.001^{\mathrm{a}}$ \\
\hline Triptan use & 17 & 12 & 0.495 \\
\hline Intra-arterial vasodilator therapy & 22 & 1 & $<0.001^{\mathrm{a}}$ \\
Length of stay, d & $14 \pm 10$ & $8 \pm 8$ & $0.004^{\mathrm{a}}$ \\
\hline Discharge mRS score 0-3 & 61 & 96 & $<0.001^{\mathrm{a}}$ \\
\hline Discharge mRS score 4-5 & 22 & 5 & $<0.001^{\mathrm{a}}$ \\
\hline Discharge mRS score 6 (death) & 17 & 0 & $<0.001^{\mathrm{a}}$
\end{tabular}

Abbreviations: ICA = internal carotid artery; $\mathrm{mRS}=$ modified Rankin Scale score; PRES = posterior reversible encephalopathy syndrome; $\mathrm{SAH}=$ subarachnoid hemorrhage.

Values are mean \pm SD or \%.

a Significant.

of prior serotonergic antidepressant use $(56 \%$ vs $31 \%, p=0.015)$ and underlying depression ( $72 \%$ vs $36 \%, p=0.001)$, and had higher rates of glucocorticoid ( $56 \%$ vs $23 \%, p=0.001)$, calcium channel blocker $(72 \%$ vs $53 \%, p=0.07)$, and intra-arterial vasodilator $(12 \%$ vs $3 \%, p=0.08)$ but not triptan therapy ( $8 \%$ vs $14 \%, p=0.33$ ). However, there were no significant differences in final lesion types or discharge clinical outcome.

Results of the exploratory multivariable regression analyses are shown in table 3. Glucocorticoid treatment proved to be a strong independent predictor of all types of worsening. Infarction on baseline imaging predicted poor outcome, but not clinical or radiologic worsening. Inta-arterial vasodilator therapy independently predicted clinical worsening and poor discharge outcome. Serotonergic antidepressant use predicted clinical and angiographic worsening. Age and sex did not prove to be independent predictors of any type of worsening or discharge outcome.

Based on these results, we investigated the relationship and mechanisms of treatment-associated clinical worsening and poor discharge outcome. Glucocorticoids were administered to 46 (28\%) patients. Thirtythree patients $(72 \%)$ received glucocorticoids due to consideration of PACNS or to treat RCVS, and the rest for a variety of reasons (e.g., contrast allergy, asthma). Different forms were used: IV methylprednisolone followed by oral prednisone $(\mathrm{n}=17)$, IV methylprednisolone alone ( $n=8)$, oral prednisone alone $(n=15)$, IV dexamethasone $(\mathrm{n}=4)$, and local injection $(\mathrm{n}=2)$. The mean time from symptom onset to glucocorticoid administration was $6.6 \pm 6.4$ days (range 1-29 days). Of the 46 patients who received steroids, 17 (37\%) showed persistent clinical worsening, 2 showed clinical improvement, and 27 showed no clinical change. Of 116 patients not treated with glucocorticoids, only 6 (5\%) showed worsening $(p<0.001)$. Of the 23 patients with clinical worsening (17 with glucocorticoid use and 6 without), 14 (61\%) developed new brain lesions including 11 new infarctions.

Figure 2 shows the days from symptom onset to clinical worsening. Included are 5 patients who also received intra-arterial vasodilator treatment. Glucocorticoid treatment preceded clinical worsening by $\leq 2$ days in all except 2 patients (one previously published ${ }^{19}$ ) who had both initiated treatment 6 days prior to worsening. Baseline parenchymal imaging was normal in 35\% of glucocorticoid-treated patients whereas all nontreated patients had abnormal baseline scans $(p=0.14)$. There was no significant difference in the angiographic severity scores between patients treated and not treated with glucocorticoids $(p=0.33)$. Among patients with clinical worsening, $47 \%$ of the glucocorticoid-treated group had discharge mRS 4-6, vs $17 \%$ in the nontreated group ( $p=0.3$ ).

Intra-arterial vasodilator treatment was administered to 7 patients, including 5 who received concomitant glucocorticoids (figure 2). Three received nicardipine, 
Table 2 Radiologic worsening in reversible cerebral vasoconstriction syndrome

\begin{tabular}{|c|c|c|c|c|c|}
\hline Variable & $\begin{array}{l}\text { No new } \\
\text { lesion }(n=113)\end{array}$ & $\begin{array}{l}\text { Any new } \\
\text { lesion }(n=44)\end{array}$ & p Value & $\begin{array}{l}\text { New infarct } \\
(n=27)\end{array}$ & p Value \\
\hline Age, y & $42 \pm 13$ & $47 \pm 11$ & 0.035 & $47 \pm 11$ & 0.096 \\
\hline Female & 73 & 91 & $0.013^{a}$ & 89 & 0.125 \\
\hline Caucasian & 88 & 70 & $0.004^{a}$ & 78 & 0.170 \\
\hline Hispanic & 9 & 25 & $0.008^{a}$ & 19 & 0.279 \\
\hline Prior hypertension & 35 & 46 & 0.204 & 48 & 0.213 \\
\hline Prior depression & 43 & 39 & 0.590 & 48 & 0.309 \\
\hline Prior migraine & 38 & 46 & 0.395 & 41 & 0.943 \\
\hline Serotonergic antidepressants & 35 & 36 & 0.910 & 44 & 0.296 \\
\hline Illicit drugs & 20 & 23 & 0.743 & 30 & 0.171 \\
\hline Postpartum state & 11 & 9 & 0.776 & 4 & 0.221 \\
\hline Idiopathic & 28 & 25 & 0.675 & 23 & 0.508 \\
\hline Recurrent thunderclap headaches & 74 & 73 & 0.837 & 63 & 0.156 \\
\hline Focal neurologic deficits & 29 & 70 & $<0.001^{a}$ & 78 & $<0.001^{a}$ \\
\hline \multicolumn{6}{|l|}{ Initial imaging } \\
\hline No lesion & 35 & 21 & 0.086 & 19 & 0.135 \\
\hline Infarct & 21 & 39 & $0.026^{a}$ & 59 & $<0.001^{a}$ \\
\hline Parenchymal hemorrhage & 12 & 11 & 0.980 & 4 & 0.164 \\
\hline Convexal SAH & 35 & 30 & 0.486 & 19 & 0.066 \\
\hline Vasogenic edema (PRES) & 24 & 32 & 0.310 & 22 & 0.613 \\
\hline \multicolumn{6}{|l|}{ Final imaging } \\
\hline Infarct & 21 & 64 & $<0.001^{a}$ & 100 & $<0.001^{a}$ \\
\hline Parenchymal hemorrhage & 12 & 16 & 0.457 & 7 & 0.361 \\
\hline Convexal SAH & 35 & 46 & 0.244 & 33 & 0.566 \\
\hline Vasogenic edema (PRES) & 24 & 39 & 0.065 & 26 & 0.790 \\
\hline Vasoconstriction score & $0.73 \pm 0.8$ & $1.01 \pm 0.4$ & $0.017^{a}$ & $1.03 \pm 0.5$ & $0.042^{a}$ \\
\hline Intracranial ICA involved & 11 & 27 & $0.015^{a}$ & 37 & $0.001^{a}$ \\
\hline Middle cerebral artery involved & 86 & 96 & 0.084 & 93 & 0.453 \\
\hline Anterior cerebral artery involved & 82 & 91 & 0.166 & 93 & 0.202 \\
\hline Posterior cerebral artery involved & 77 & 86 & 0.175 & 82 & 0.764 \\
\hline Vertebral or basilar arteries involved & 45 & 61 & 0.067 & 67 & 0.052 \\
\hline Calcium channel blocker therapy & 3 & 61 & 0.349 & 70 & 0.086 \\
\hline Cyclophosphamide therapy & 3 & 12 & $0.026^{a}$ & 11 & 0.140 \\
\hline Glucocorticoid use & 18 & 55 & $<0.001^{a}$ & 63 & $<0.001^{a}$ \\
\hline Triptan use & 13 & 14 & 0.952 & 8 & 0.317 \\
\hline Interventional dilatators therapy & 3 & 9 & 0.099 & 15 & $0.018^{a}$ \\
\hline Length of stay, $d$ & $7 \pm 6$ & $16 \pm 11$ & $<0.001^{a}$ & $19 \pm 12$ & $<0.001^{a}$ \\
\hline Discharge $\mathrm{mRS}$ score $0-3$ & 97 & 76 & $<0.001^{a}$ & 63 & $<0.001^{a}$ \\
\hline Discharge mRS score 4-5 & 4 & 16 & $<0.001^{a}$ & 22 & $<0.001^{a}$ \\
\hline Discharge mRS score 6 (death) & 0 & 9 & $0.006^{a}$ & 15 & $<0.001^{a}$ \\
\hline
\end{tabular}

Abbreviations: ICA = internal carotid artery; $\mathrm{mRS}$ = modified Rankin Scale score; PRES = posterior reversible encephalopathy syndrome; $\mathrm{SAH}=$ subarachnoid hemorrhage.

Values are mean \pm SD or \%.

a Significant. 
Table 3 Exploratory multivariable models: Predictors of worsening and poor outcome

Outcome variable

OR $(95 \% \mathrm{Cl})$

p Value

Clinical worsening $(n=23)$

Model 1

Female sex

Infarction on baseline imaging

Prior hypertension

Serotonergic antidepressant use

Model 2

Female sex

Infarction on baseline imaging

Glucocorticoids

IA vasodilator

Radiologic worsening $(n=44)$

Age, y

Female sex

Hispanic ethnicity

Focal neurologic deficit at onset

Infarction on baseline imaging

Glucocorticoids

Angiographic worsening $(n=25)$

Female sex

Serotonergic antidepressant use

Glucocorticoids

Calcium channel blockers

Poor outcome (mRS $>3, n=15)$

Model 1

Female sex

Infarction on baseline imaging

Serotonergic antidepressant use

Model 2

Female sex

$0.67(0.18-2.64)$

8.55 (2.30-31.8)

4.23 (1.20-14.9)

$9.77(1.48-64.4)$

0.291

0.087

0.014

0.022

0.067

0.384

$<0.001$

0.015

0.203

0.519

0.078

0.002

0.524

0.001

0.587

0.041

0.004

0.122

Infarction on baseline imaging

Glucocorticoids

IA vasodilator
Abbreviations: $\mathrm{CI}=$ confidence interval; $\mathrm{IA}=$ intra-arterial; $\mathrm{OR}=$ odds ratio.

2 verapamil, 2 milrinone, and 1 papaverine. The angiographic response (prompt vasodilation) was uniform but often ill-sustained. ${ }^{19}$ Four continued to deteriorate or showed no clinical improvement, and 3 improved. Finally, triptans were used in 21 (13\%) patients to treat the onset headache. Four reported acute exacerbation of pain, 1 had seizures, ${ }^{12}$ and 6 (29\%) showed subsequent clinical worsening.

DISCUSSION In this study, we provide important information about the frequency and characteristics of worsening in RCVS. There was a significant correlation among persistent clinical worsening, new radiologic lesions, and initial angiographic progression. Our results provide clarity on the influence of several baseline variables on worsening. Most importantly, we show that worsening often occurs after glucocorticoid initiation, and that therapeutic strategies such as calcium channel blockers and triptans are usually ineffective in treating headache or preventing clinical or radiologic complications. The effects of intraarterial vasodilator therapy seem variable; its role is discussed below.

Our results have profound implications on the existing approach to RCVS management. Although the vast majority of patients with RCVS have a selflimited course lasting days to weeks with benign outcome $e^{1-4,6,7}$ and good long-term prognosis, ${ }^{20,21}$ the presence of widespread, severe angiographic abnormalities often prompts the administration of calcium channel blockers to address headaches or vasospasm, or pharmacologically induced hypertension in an attempt to improve cerebral perfusion. Glucocorticoids are often administered due to misdiagnosis as PACNS, or due to the fear of missing PACNS while awaiting angiographic reversibility to confirm the diagnosis of RCVS. ${ }^{6,19}$ While the authors have never treated RCVS with glucocorticoids, patients in this study were exposed prior to transfer or at our own hospital due to diagnostic uncertainties or for systemic indications (e.g., contrast allergy). Intra-arterial vasodilator infusion is frequently offered even in clinically stable patients to treat the angiographic findings; some centers advocate using this interventional procedure for early diagnosis based on the angiographic response to vasodilator infusion. ${ }^{16,22}$ We have recently shown that the diagnosis can be made with alacrity based on bedside clinical and brain imaging features alone $e^{7,23}$ and that our criteria have nearly $100 \%$ specificity in distinguishing RCVS from PACNS. ${ }^{7}$ Hence, the use of potentially risky invasive procedures cannot be justified for diagnostic purposes, ${ }^{24}$ particularly since the outcome is usually excellent. We show that in the event of diagnostic uncertainty or in patients with clinical stability or with minor/nonprogressive deficits, it is important to withhold glucocorticoids and catheter-based interventions. Symptomatic pain management, the removal of vasoconstrictive precipitants, and strategies to avoid the Valsalva maneuver (which can trigger recurrent thunderclap headaches) are typically adequate while awaiting the natural resolution of headache and vasoconstriction. ${ }^{25}$

The management of patients with clinical progression remains challenging. Prior studies have shown that $16 \%-34 \%$ of patients develop transient neurologic deficits, including seizures, but fewer than $10 \%$ develop mild to moderate permanent deficits and 


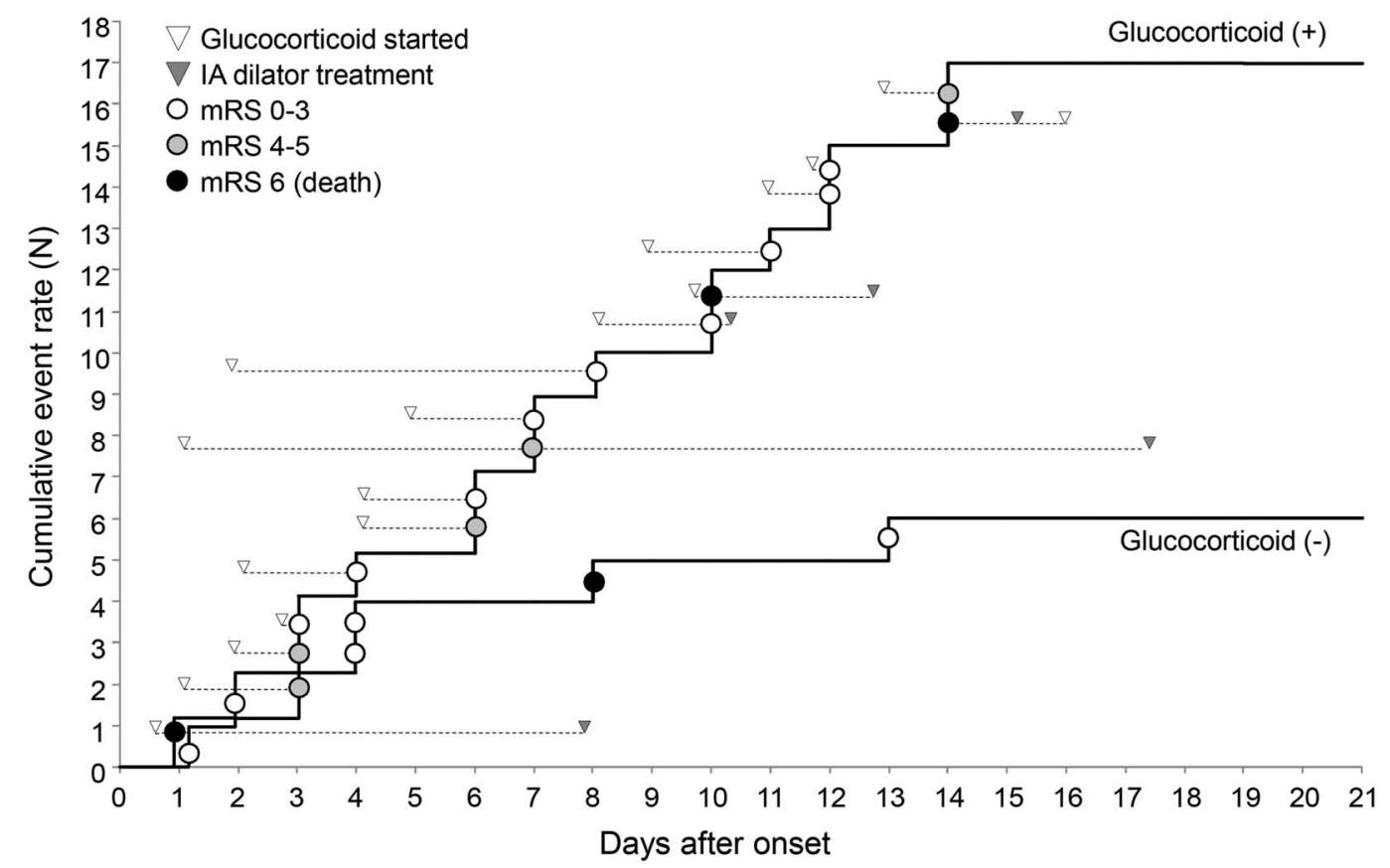

Cumulative event rate curves show the time of clinical progression with or without glucocorticoid and IA vasodilator treatment. Circles along the curves depict individual patients. Circles are colored according to the patient's eventual discharge modified Rankin Scale scores (mRS) as follows: white, mRS 0-3; gray, mRS 4-5; black, mRS 6 (death). White arrowheads reflect the day after reversible cerebral vasoconstriction syndrome (RCVS) onset when glucocorticoids were initiated. Gray arrowheads reflect the day after RCVS onset when IA vasodilator treatment was administered. The length of the dotted lines connecting arrowheads to circles reflects the interval between treatment and worsening.

only a minority show relentless progression that can be fatal. ${ }^{2,6,10,19,26}$ Ideally, specific treatments should be targeted to patients with high likelihood for poor outcome. At present it is challenging to identify such patients and intervene before significant irreversible damage occurs. Our analysis focused on predicting poor discharge outcome, unlike prior studies that investigated predictors of any clinical worsening (transient or permanent). ${ }^{10}$ We show that baseline infarction and glucocorticoid exposure are independent predictors of poor outcome. Patients with these features should be carefully monitored, preferably in an intensive care setting. Most such patients will declare clinical stability or improve in a few days. In the event of definite clinical or radiologic progression, glucocorticoids should be withheld but intra-arterial vasodilator treatment may still be considered on the basis of anecdotal case reports. ${ }^{17,27-29}$ Substantial clinical experience and judgment is required to determine the appropriate threshold for intervention.

In this study, some factors proved to be independent predictors of clinical or radiologic or angiographic worsening, but not poor clinical outcome. For example, serotonergic antidepressant use predicted clinical and angiographic worsening but exposed patients did not have significant disability at the time of discharge. This may suggest that their effects are mild, or that cessation (due to their acknowledged role as triggers) prevented any sustained effects. These findings are consistent with our prior study showing that selective serotonin reuptake inhibitors worsen vasospasm but not clinical outcome in patients with aneurysmal subarachnoid hemorrhage, ${ }^{13}$ and another study where serotonergic antidepressants tended to predict symptomatic vasospasm but not clinical or radiologic complications after aneurysmal SAH. ${ }^{14}$ Similarly, we found that triptans, which have serotonergic effects, showed no statistically significant effect on worsening but approximately half the patients developed new neurologic symptoms (increased pain, seizures, new deficits) after treatment. These data affirm the importance of withholding serotonergic agents in RCVS. ${ }^{12}$ Whether they can be resumed after RCVS resolution is not clear. Further, these data support a pathophysiologic role of serotonergic mechanisms in triggering RCVS.

As in a prior study, ${ }^{10}$ we found no association between clinical/radiologic worsening and factors such as age, sex, migraine, brain hemorrhage, or PRES on baseline imaging. In addition, these factors were not associated with poor outcome (table 3). However, prior hypertension was associated with clinical worsening. We speculate that chronically hypertensive patients had greater susceptibility to cerebral ischemia due to underlying small-vessel disease, but did not 
develop infarction due to the dynamic changes in arterial caliber. Finally, it is important to note that cSAH and normal parenchymal imaging were associated with less clinical and radiologic worsening, and better outcomes. Patients with these features may not require intensive monitoring or prolonged observation.

Our results provide insights about the mechanisms of worsening in RCVS. Patients with clinical worsening had new brain lesions (mainly infarcts), and those with angiographic progression had more clinical worsening as well as new infarcts and edema (PRES). These data confirm that worsening occurs due to angiographic progression, primarily due to new nonhemorrhagic lesions. Importantly, our study shows that hemorrhagic RCVS is not associated with poor outcome, unlike other conditions associated with lobar hemorrhage. Iatrogenic factors appear to play a major role. Glucocorticoids clearly do not prevent worsening, and may in fact induce worsening by potentiating the effects of vasoconstrictors such as norepinephrine, angiotensin II, endothelin, and others, as well as their direct actions on vascular smooth muscle cells. ${ }^{30}$ The time course of such effects (hours to days) ${ }^{30}$ correlates with our observation of worsening occurring within 2-6 days after treatment initiation in RCVS. These results are consistent with recent analyses showing a deleterious effect of glucocorticoid use (especially recent initiation) on stroke outcome. $^{31}$

The relatively large sample size, the availability of serial imaging data, and the correlation between different outcomes lends confidence to our results. The retrospective design is a limitation. The identified predictors could be validated in existing prospective datasets; however, prospective trials investigating the effects of glucocorticoids, serotonergic agents, or other potential predictors of poor outcome would be unethical. Due to the retrospective design, there was variability in the modality and timing of followup imaging studies and clinical selection bias in obtaining imaging follow-up. Though larger than other cohort studies, the number of exposures and outcome events were limited; hence we had to create multiple regression models for exploratory analyses. Finally, 30 patients had probable RCVS based on previously published criteria. ${ }^{1}$ There was no significant difference in the percentage of patients with clinical, radiologic, or angiographic worsening between these and the remaining 132 patients (data not shown). Further, all 162 patients had one or more recently identified feature with $98 \%-100 \%$ specificity for the diagnosis of RCVS (e.g., recurrent thunderclap headaches, or a single thunderclap headache combined with normal neuroimaging, border zone infarcts, or vasogenic edema), ${ }^{7}$ so using contemporary criteria the diagnosis is secure in our entire cohort.
Although prior publications have shown an association between glucocorticoids and poor outcome, ${ }^{6,7}$ these agents continue to be administered. The reasons are unclear, but may be related to underappreciation of prior results or skepticism because of possible selection bias - an issue we have directly addressed here. We are encouraged that the frequency of glucocorticoid treatment in our cohort has decreased from 32\% to $19 \%$ after our 2011 publication. ${ }^{6}$ We hope our results will inform the treating clinician about the appropriate management of RCVS. The focus should be on accurate bedside diagnosis using contemporary criteria, ${ }^{7}$ after which less is more and primum non nocere should be the guiding principles.

\section{AUTHOR CONTRIBUTIONS}

A.B.S. and M.A.T. conceived and designed the study, analyzed the data, drafted the text, and prepared the figures. Both authors participated in data acquisition and manuscript revisions and approved the final version.

\section{STUDY FUNDING}

No targeted funding reported.

\section{DISCLOSURE}

A. Singhal has served as a medical expert witness and has received honoraria from the American Academy of Neurology, Medlink, Inc., and UptoDate. M. Topcuoglu reports no disclosures relevant to the manuscript. Go to Neurology.org for full disclosures.

Received April 5, 2016. Accepted in final form August 23, 2016.

\section{REFERENCES}

1. Calabrese LH, Dodick DW, Schwedt TJ, Singhal AB. Narrative review: reversible cerebral vasoconstriction syndromes. Ann Intern Med 2007;146:34-44.

2. Ducros A, Boukobza M, Porcher R, Sarov M, Valade D, Bousser MG. The clinical and radiological spectrum of reversible cerebral vasoconstriction syndrome: a prospective series of 67 patients. Brain 2007;130:3091-3101.

3. Chen SP, Fuh JL, Wang SJ, et al. Magnetic resonance angiography in reversible cerebral vasoconstriction syndromes. Ann Neurol 2010;67:648-656.

4. Ducros A, Fiedler U, Porcher R, Boukobza M, Stapf C, Bousser MG. Hemorrhagic manifestations of reversible cerebral vasoconstriction syndrome: frequency, features, and risk factors. Stroke 2010;41:2505-2511.

5. Topcuoglu MA, Singhal AB. Hemorrhagic reversible cerebral vasoconstriction syndrome: features and mechanisms. Stroke 2016;47:1742-1747.

6. Singhal AB, Hajj-Ali RA, Topcuoglu MA, et al. Reversible cerebral vasoconstriction syndromes: analysis of 139 cases. Arch Neurol 2011;68:1005-1012.

7. Singhal AB, Topcuoglu MA, Fok JW, et al. Reversible cerebral vasoconstriction syndromes and primary angiitis of the central nervous system: clinical, imaging, and angiographic comparison. Ann Neurol 2016;79: 882-894.

8. Hajj-Ali RA, Furlan A, Abou-Chebel A, Calabrese LH. Benign angiopathy of the central nervous system: cohort of 16 patients with clinical course and long-term followup. Arthritis Rheum 2002;47:662-669.

9. Calabrese LH, Gragg LA, Furlan AJ. Benign angiopathy: a distinct subset of angiographically defined primary 
angiitis of the central nervous system. J Rheumatol 1993; 20:2046-2050.

10. Katz BS, Fugate JE, Ameriso SF, et al. Clinical worsening in reversible cerebral vasoconstriction syndrome. JAMA Neurol 2014;71:68-73.

11. Robert T, Kawkabani Marchini A, Oumarou G, Uske A. Reversible cerebral vasoconstriction syndrome identification of prognostic factors. Clin Neurol Neurosurg 2013; 115:2351-2357.

12. Singhal AB, Caviness VS, Begleiter AF, Mark EJ, Rordorf G, Koroshetz WJ. Cerebral vasoconstriction and stroke after use of serotonergic drugs. Neurology 2002;58:130-133.

13. Singhal AB, Topcuoglu MA, Dorer DJ, Ogilvy CS, Carter BS, Koroshetz WJ. SSRI and statin use increases the risk for vasospasm after subarachnoid hemorrhage. Neurology 2005;64:1008-1013.

14. Young JB, Singh TD, Rabinstein AA, Fugate JE. SSRI/SNRI use is not associated with increased risk of delayed cerebral ischemia after aSAH. Neurocrit Care 2016;24:197-201.

15. Zuber M, Touze E, Domigo V, Trystram D, Lamy C, Mas JL. Reversible cerebral angiopathy: efficacy of nimodipine. J Neurol 2006;253:1585-1588.

16. Linn J, Fesl G, Ottomeyer C, et al. Intra-arterial application of nimodipine in reversible cerebral vasoconstriction syndrome: a diagnostic tool in select cases? Cephalalgia 2011;31:1074-1081.

17. Farid H, Tatum JK, Wong C, Halbach VV, Hetts SW. Reversible cerebral vasoconstriction syndrome: treatment with combined intra-arterial verapamil infusion and intracranial angioplasty. AJNR Am J Neuroradiol 2011;32: E184-E187.

18. Bartynski WS. Posterior reversible encephalopathy syndrome, part 1: fundamental imaging and clinical features. AJNR Am J Neuroradiol 2008;29:1036-1042.

19. Singhal AB, Kimberly WT, Schaefer PW, Hedley-Whyte ET. Case records of the Massachusetts General Hospital: case 8-2009: a 36-year-old woman with headache, hypertension, and seizure 2 weeks post partum. N Engl J Med 2009;360:1126-1137.
20. Chen SP, Fuh JL, Lirng JF, Wang YF, Wang SJ. Recurrence of reversible cerebral vasoconstriction syndrome: a long-term follow-up study. Neurology 2015;84:1552-1558.

21. John S, Singhal AB, Calabrese L, et al. Long-term outcomes after reversible cerebral vasoconstriction syndrome. Cephalalgia 2015;36:387-394.

22. Kass-Hout T, Kass-Hout O, Sun $\mathrm{CH}$, et al. A novel approach to diagnose reversible cerebral vasoconstriction syndrome: a case series. J Stroke Cerebrovasc Dis 2015;24: e31-37.

23. Muehlschlegel S, Kursun O, Topcuoglu MA, Fok J, Singhal $\mathrm{AB}$. Differentiating reversible cerebral vasoconstriction syndrome with subarachnoid hemorrhage from other causes of subarachnoid hemorrhage. JAMA Neurol 2013;70:1254-1260.

24. Singhal AB. Diagnostic challenges in RCVS, PACNS, and other cerebral arteriopathies. Cephalalgia 2011;31:10671070.

25. Singhal AB. Reversible cerebral vasoconstriction syndromes: what the cardiologist should know. Curr Treat Options Cardiovasc Med 2014;16:290.

26. Fugate JE, Wijdicks EF, Parisi JE, et al. Fulminant postpartum cerebral vasoconstriction syndrome. Arch Neurol 2012;69:111-117.

27. Bouchard M, Verreault S, Gariepy JL, Dupre N. Intraarterial milrinone for reversible cerebral vasoconstriction syndrome. Headache 2009;49:142-145.

28. Elstner M, Linn J, Muller-Schunk S, Straube A. Reversible cerebral vasoconstriction syndrome: a complicated clinical course treated with intra-arterial application of nimodipine. Cephalalgia 2009.

29. Ringer AJ, Qureshi AI, Kim SH, Fessler RD, Guterman LR, Hopkins LN. Angioplasty for cerebral vasospasm from eclampsia. Surg Neurol 2001;56:373-378; discussion 378-379.

30. Ullian ME. The role of corticosteriods in the regulation of vascular tone. Cardiovasc Res 1999;41:55-64.

31. Sundboll J, Horvath-Puho E, Schmidt M, et al. Preadmission use of glucocorticoids and 30-day mortality after stroke. Stroke 2016;47:829-835.

\section{Share Your Artistic Expressions in Neurology 'Visions'}

AAN members are urged to submit medically or scientifically related artistic images, such as photographs, photomicrographs, and paintings, to the "Visions" section of Neurology ${ }^{\circledR}$. These images are creative in nature, rather than the medically instructive images published in the NeuroImages section. The image or series of up to six images may be black and white or color and must fit into one published journal page. Accompanying description should be 100 words or less; the title should be a maximum of 96 characters including spaces and punctuation.

Learn more at www.aan.com/view/Visions, or upload a Visions submission at submit.neurology.org. 